\title{
Molecular, clinical, and muscle studies in myotonic dystrophy type 1 (DM1) associated with novel variant CCG expansions
}

\author{
Massimo Santoro $\cdot$ Marcella Masciullo $\cdot$ Roberta Pietrobono $\cdot$ Giulia Conte • \\ Anna Modoni · Maria Laura E. Bianchi · Valentina Rizzo • Maria Grazia Pomponi • \\ Giorgio Tasca · Giovanni Neri $\cdot$ Gabriella Silvestri
}

Received: 24 September 2012/Revised: 22 November 2012/ Accepted: 28 November 2012

(C) Springer-Verlag Berlin Heidelberg 2012

\begin{abstract}
We assessed clinical, molecular and muscle histopathological features in five unrelated Italian DM1 patients carrying novel variant pathological expansions containing CCG interruptions within the $3^{\prime}$-end of the CTG array at the $D M P K$ locus, detected by bidirectional triplet primed PCR (TP-PCR) and sequencing. Three patients had a negative DM1 testing by routine long-range PCR; the other two patients were identified among 100 unrelated DM1 cases and re-evaluated to estimate the prevalence of variant expansions. The overall prevalence was $4.8 \%$ in our study cohort. There were no major clinical differences between variant and non-variant DM1 patients, except for cognitive involvement. Muscle RNA-FISH, immunofluorescence for MBNL1 and RT-PCR analysis documented the presence of ribonuclear inclusions, their co-localization
\end{abstract}

Electronic supplementary material The online version of this article (doi:10.1007/s00415-012-6779-9) contains supplementary material, which is available to authorized users.

M. Santoro - G. Tasca

Fondazione Don Carlo Gnocchi, ONLUS, Milan, Italy

M. Masciullo · G. Conte · A. Modoni ·

M. L. E. Bianchi · V. Rizzo · G. Silvestri ( $\square)$ Department of Geriatrics, Neuroscience and Orthopedics, Center for Neuromuscular Disorders, Catholic University of Sacred Heart, L.go F. Vito, 1, Rome, Italy

e-mail: gsilvestri@rm.unicatt.it

M. Masciullo

Department of Neuromotor Rehabilitation,

IRCCS San Raffaele Pisana, Rome, Italy

R. Pietrobono · M. G. Pomponi - G. Neri

Department of Medical Genetics,

Catholic University of Sacred Heart, Rome, Italy with MBNL1, and an aberrant splicing pattern involved in DM1 pathogenesis, without any obvious differences between variant and non-variant DM1 patients. Therefore, this study shows that the CCG interruptions at the $3^{\prime}$-end of expanded DMPK alleles do not produce qualitative effects on the RNA-mediated toxic gain-of-function in DM1 muscle tissues. Finally, our results support the conclusion that different patterns of CCG interruptions within the CTG array could modulate the DM1 clinical phenotype, variably affecting the mutational dynamics of the variant repeat.

Keywords Steinert's disease - Myotonic dystrophy type $1 \cdot$ Variant expansions

\section{Introduction}

Myotonic dystrophy type 1 (DM1, dystrophia myotonica, Steinert's disease; OMIM 160900) is a multisystem disorder affecting skeletal muscle and several other tissues, including the heart, brain, smooth muscle, eye and the endocrine system $[1,2]$.

The molecular defect underlying DM1 consists of an abnormal expansion of a polymorphic CTG-repeat tract in the $3^{\prime}$ untranslated region (UTR) of the DMPK gene, located on chromosome 19q13.2-q13.3 [3-5]. Normal alleles at the DM1 locus contain 5 to 35 CTG repeats, whereas pathogenic alleles range from 50 to more than 1,000 repeats $[3,5,6]$.

While the wild-type CTG tract is relatively stable upon transmission, both intermediate premutated alleles (range 35-49 CTG repeats, mostly identified in unaffected DM1 parents) and full-range expanded alleles (transmitted from affected individuals) are biased toward expansion [7-9]. 
Similar to other triplet repeats expansion diseases (TREDs), the occurrence of a slipped-strand mispairing within the repetitive sequences during a broad range of DNA metabolic processes represents the most likely mechanism underlying the mutational dynamics of DM1 expanded repeats [10]. DM1 is characterized by genetic "anticipation" through subsequent affected generations, a phenomenon associated with an increase in the number of repeats during intergenerational transmission [1, 2]. Moreover, the expanded CTG tract in DM1 shows somatic instability leading to somatic mosaicism, which can partially explain both the phenotypic variability among DM1 patients and the lack of clear-cut correlations between the number of CTG repeats in leukocytes and the severity of some clinical manifestations [2].

Recently, pathological "variant" expansions containing sequence interruptions (CCG, CTC, and GGC repeats) interspersed within the $3^{\prime}$-end of the CTG array have been reported in DM1 patients by two distinct studies, with an estimated prevalence of 3-5\% of cases [11, 12].

As for other TREDs [13, 14], these studies documented that the presence of interruptions within the CTG array at the DM1 locus would stabilize the variant expanded alleles during intergenerational transmission [11, 12]. They also highlighted the recurrence of atypical symptoms in affected individuals of some DM1 families carrying "variant" expansions [11]. This suggests that interruptions in the regular primary structure of the CTG expansion could modulate the clinical phenotype, either by increasing DNA strand stability during cell divisions, or by producing conformational changes of the variant RNA species with effects on their toxic gain-of-function [11, 12].

In order to clarify these issues, we performed a detailed clinical and muscle characterization in five unrelated Italian DM1 patients carrying novel "variant" expansions, diagnosed in our study cohort including 103 DM1 families.

Analysis for variant CTG expansions performed on DNA extracted from leukocytes included bidirectional TPPCR followed by sequencing studies $[12,15,16]$.

The prevalence of variant expansions estimated in our DM1 population was compared to literature data. Detailed clinical features were assessed in the five variant DM1 patients.

Finally, in three patients we performed morphological and molecular studies on muscle biopsies, including RNA fluorescence in situ hybridization (RNA-FISH) combined with immunofluorescence (IF) for MBNL1and RT-PCR studies for INSR, TNNT2, RYRI, and CLCN1 RNAs, to evaluate if the interruptions within the CTG array would produce any effect on the RNA-mediated pathogenesis at the muscle tissue level in DM1.

\section{Materials and methods}

The study design fulfilled the guidelines of the Ethical Board of our Institution.

Patients

The clinical-diagnostic assessment included:

1. Neurological evaluation and scoring of the severity of skeletal muscle involvement by means of the Muscular Impairment Rating Scale (MIRS) [17].

2. Assessment of glucose metabolism, electrolytes, liver and kidney function, serum electrophoresis, thyroid and sexual hormones determination.

3. Standard EMG and nerve conduction studies with bilateral examination of sural and tibial nerves with F-waves studies.

4. Neuropsychological examination, as previously reported [18]. Performances were considered either pathological or normal according to cut-off scores obtained by standardization studies, which also provided age and educational level-adjusted scores.

5. Pelvic and lower limb muscles MRI, performed by a 1.5-T system (Siemens Vision, Siemens, Erlangen, Germany) equipped with a phase-array multicoil. The studies included T1-weighted spin-echo (SE) T2weighted SE and fat-suppressed T2-weighted SE sequences in both axial and coronal sections.

6. Standard cardiologic evaluation including basal ECG recording, 24-h-Holter monitoring and echocardiography. Recommendations for cardiac electrophysiological studies (SEF) were given to patients in accordance to the inclusion criteria of the RAMYD study [19].

7. Lung function parameters, including forced vital and total lung capacities assessed by spirometric evaluation. A chest X-ray and a nocturnal pulse-oximetry were also performed in all patients.

8. An ophthalmologic evaluation including dilated fundus and slit-lamp examinations, and tone audiometry for hearing function assessment.

Clinical and diagnostic findings of patients $1-5$ are summarized in Table 1. Patients' clinical data are reported in the supplementary file, and neuropsychological tests scores in the Table S1.

Patients 1-3 had previously tested negative for DM1. We repeated DM1 testing by routine long-range (LR) PCR/ Southern blotting and the results were negative, with all patients apparently being homozygotic for a wild-type allele (Fig. 1) [20]. DM2 testing was negative too (data not shown). 


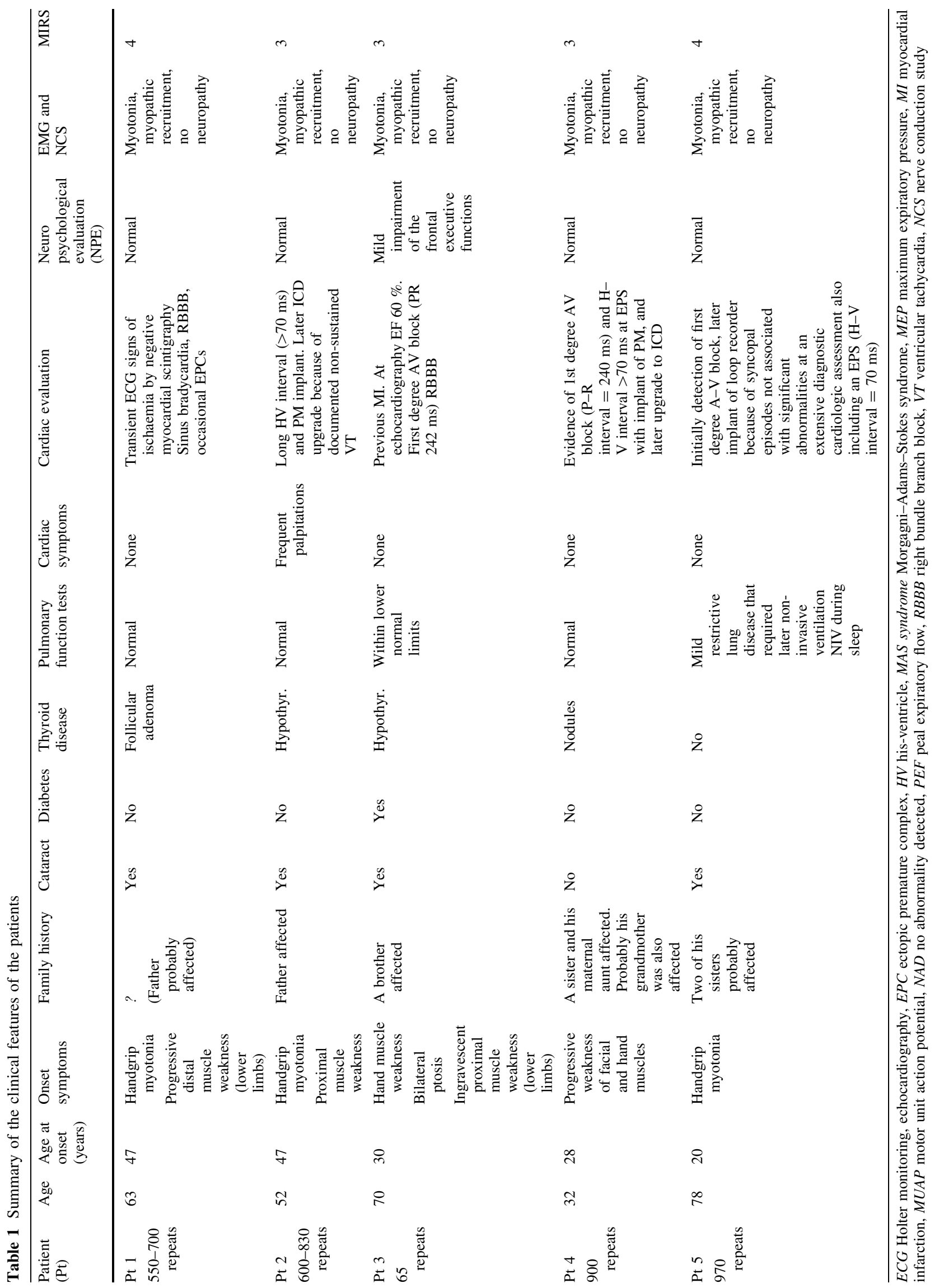




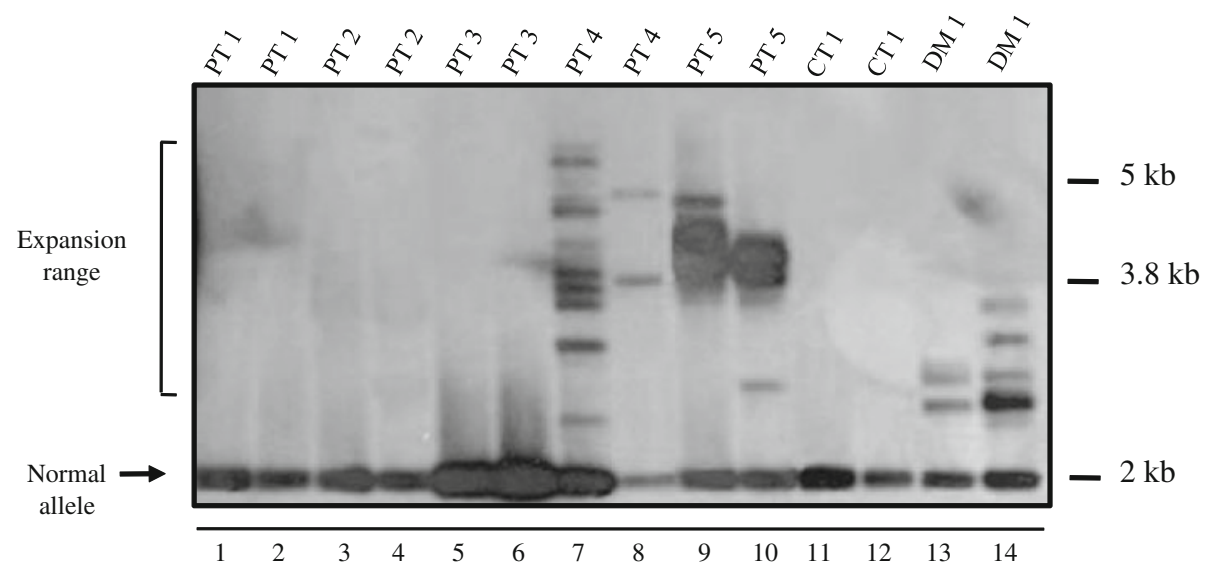

Fig. 1 LR-PCR/Southern blot. For each sample, reactions were performed in duplicate: lanes $1-6=$ pts $1-3$; lanes $7-10=$ pts $4-5$; lanes $11-12=$ control; lanes $13-14=$ DM1 patient. Fragments corresponding to wild-type alleles show a molecular weight $(\mathrm{MW})$

Patients 4 and 5 had already received a genetic diagnosis of DM1 by LR PCR/Southern blotting at our department (Fig. 1).

\section{DNA extraction and LR-PCR}

A written informed consent for DM1 molecular testing was obtained from all patients. Genomic DNA was extracted from peripheral blood lymphocytes and muscle biopsies by the Wizard Genomic DNA Purification Kit (Promega, Woods Hollow Road Madison, USA). LR-PCR/Southern blot, routinely used for DM1 molecular diagnosis was performed as described previously [20].

\section{Bidirectional triplet primed PCR}

Bidirectional TP-PCR was performed using "Expand Long Template PCR System Kit" (Roche Diagnostics, Mannheim, Germany) which includes a combination of two thermostable Taq polymerases. A negative and a positive control (DM1 with positive long-range testing) were also included in each set of reactions.

For the "forward" reaction 50-150 ng of DNA were amplified in a reaction volume of $25 \mu$ l, using 2.5 units of DNA polymerase mix, Buffer $11 \mathrm{X}, 0.8 \mu \mathrm{M}$ of primers 6-FAM labeled-DM1 F 5'-GCT CGAAGGGTCCTTGTAG CCG-3', P3 (tail) 5'-TACGCATCCCAGTTTGAGACG-3' and $0.08 \mu \mathrm{M}$ of the primer DM1/CAG R $5^{\prime}$-tacgcatcccag tttgagacgCAGCAGCAGCAGCAGCAGCAGCAG-3',

Betaine 1.3 M, $0.4 \mu \mathrm{M}$ of each dATP, dCTP, dTTP, and dGTP. Cycling conditions were: $10 \mathrm{~min} / 98^{\circ} \mathrm{C}, 15 \mathrm{~s} / 94{ }^{\circ} \mathrm{C}, 30 \mathrm{~s} /$ $62{ }^{\circ} \mathrm{C}, 5 \mathrm{~min} / 68^{\circ} \mathrm{C}$ for 10 cycles; $15 \mathrm{~s} / 94{ }^{\circ} \mathrm{C}, 30 \mathrm{~s} / 62{ }^{\circ} \mathrm{C}$, $5 \mathrm{~min} / 68{ }^{\circ} \mathrm{C}, 20 \mathrm{~s} / 68{ }^{\circ} \mathrm{C}$ for 25 cycles and $7 \mathrm{~min} / 68^{\circ} \mathrm{C}$.

Standard reverse TP-PCR was performed using the following primers: 6-FAM labeled-DM1 R 5'-CCTGCAG of about $2 \mathrm{~kb}$. The DM1 patient shows fully expanded alleles with MW between 2.8 and $3.8 \mathrm{~kb}$. Pts 1-3 show an apparent homozygosity for a wild-type allele; expanded alleles between 3.8 and $5 \mathrm{~kb}$ were also detected in pts 4 and 5 by this procedure

TTTGCCCATCCACG-3', P3 (tail) and DM1/CTG F 5'-

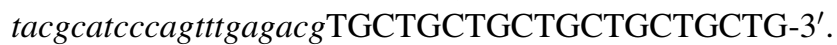

Patients showing an aberrant pattern at standard reverse TP-PCR (Supplementary Fig. 1B) were further assessed by a second round of reverse TP-PCR and subsequent sequencing studies using in the reactions primers DM1/CC F $5^{\prime}$-tacgcatcccagtttgagacgTGCTGCTGCTGCTGCTGCT GCC-3' and DM1/CCG F: $5^{\prime}$-tacgcatcceagtttgagacgTGCC GCTGCCGCTGCCGCTGCC-3', that allow to identify variant expansions containing CCG interruptions at the $3^{\prime}$ end of the CTG array at the DMPK locus [12].

For capillary electrophoresis, $2 \mu \mathrm{l}$ of the TP-PCR products were analyzed on an ABI 3130 automatic sequencer using GS500 LIZ (Applied Biosystems, Foster City, USA) as internal markers.

\section{Sequencing}

Both forward and reverse (using primer DM1 CC F) TPPCR products were purified using SureClean (Bioline, London, UK) and directly sequenced using unlabeled DM1 $\mathrm{F}$ and DM1 R respectively on an ABI 3130 automatic sequencer and BigDye ${ }^{\circledR}$ XTerminator $^{\mathrm{TM}}$ Purification Kit (Applied Biosystems, Foster City, USA).

\section{Fluorescent-PCR}

The sizing of the variant alleles was assessed on leukocytes DNA from pts 1-3 and from one typical DM1 patient by fluorescent PCR using the Expand Long Template PCR System Kit (Roche Diagnostics, Mannheim, Germany) [21, 22]. Reaction conditions included buffer one with $10 \%$ DMSO, $0.4 \mu \mathrm{M}$ of each dATP, dCTP, dTTP, and dGTP, 15 pmol of the forward primer 6-FAM labeled-MDY 1D and 15 pmol of the reverse primer MDYZ2A [20]. 
Cycling conditions were: $10 \mathrm{~min} / 98{ }^{\circ} \mathrm{C}, 15 \mathrm{~s} / 94{ }^{\circ} \mathrm{C}, 30 \mathrm{~s} /$ $62{ }^{\circ} \mathrm{C}, 5 \mathrm{~min} / 68^{\circ} \mathrm{C}$ for 10 cycles; $15 \mathrm{~s} / 94{ }^{\circ} \mathrm{C}, 30 \mathrm{~s} / 62{ }^{\circ} \mathrm{C}$, $5 \mathrm{~min} / 68{ }^{\circ} \mathrm{C}, 20 \mathrm{~s} / 68{ }^{\circ} \mathrm{C}$ for 25 cycles and $7 \mathrm{~min} / 68^{\circ} \mathrm{C}$.

$5 \mu \mathrm{l}$ of the PCR products were analyzed on ABI 3130 automatic sequencer using GS1200 LIZ (Applied Biosystems, Foster City, USA) as internal markers.

The size of expanded alleles was estimated by Southern blot analysis of fluorescent PCR products: briefly, after amplification $15 \mu \mathrm{l}$ of reaction products were separated by electrophoresis overnight at $40 \mathrm{~V}$ in $1 \%$ agarose gel. Gels were then blotted and hybridized with (CTG) $5{ }^{32} \mathrm{P}$-labeled oligonucleotide. After two washes in 3xSSC and 0.1\% SDS at $44^{\circ} \mathrm{C}$, filters were exposed to Biomax MR autoradiography films (Kodak film, Rochester, USA) for $2 \mathrm{~h}$ at $-80{ }^{\circ} \mathrm{C}$.

Muscle RNA-FISH-immunofluorescence (FISH-IF) for MBNL1

$6 \mu$ m-thick, serial transverse muscle cryostat sections from pts 1-3 and one typical DM1 patient were processed as previously described [23].

In order to get the same treatment, muscle sections from control and three patients were placed on the same slide. Muscle sections were examined using a fluorescence microscope (Olympus BH2-RFCA).

\section{Muscle RT-PCR studies}

Total RNA was extracted from muscle tissues using Trizol reagent (Invitrogen, Carlsbad, California), according to the manufacturer's instructions. The first strand cDNA was synthesized using $1 \mu \mathrm{g}$ of total RNA DNaseI treated, $100 \mathrm{ng}$ oligo(dt) ${ }_{12-18}$ primer (Invitrogen, Carlsbad, California), $0.5 \mathrm{mM}$ dNTPs and 200 units SuperScript II reverse transcriptase (Invitrogen, Carlsbad, California) at $42{ }^{\circ} \mathrm{C}$ for $1 \mathrm{~h}$.

For RT-PCR studies of INSR, TNNT2, RYRI and CLCN1 mRNAs, PCR amplification of the individual cDNA fragments was carried out using oligonucleotides previously described [24-27].

RT-PCR products were resolved on $8 \%$ non-denaturing polyacrylamide gels and stained with ethidium bromide. Densitometric analysis was carried out using the TotalLab 12.01 software (Nonlinear Dynamics Ltd., Newcastle upon Tyne, UK); the relative amount of each aberrant premRNA specie was estimated as percentage value of the aberrant isoform versus its corresponding total pre-mRNA.

\section{Results}

\section{TP-PCR analysis}

In pts1-3, routine DM1 testing by long-range PCR showed a unique band in the range of normal alleles (Fig. 1).
In order to rule out false negative results, we therefore decided to perform bidirectional TP-PCR. This analysis showed two close peaks both in the normal range in the healthy heterozygous subject (data not shown). In the canonical DM1 patient, the electropherogram showed a ladder peak in the pathological range whose height diminished gradually with increasing product size both for the forward and the reverse TP-PCR reactions (Fig. 2b).

In pts 1-3 the "forward" TP-PCR clearly documented pathologically expanded alleles similar to those ones detected in the non-variant DM1 patient (Fig. 2c, left panel). Moreover, the electropherograms of the "reverse" TP-PCR using DM1/CTG F showed an aberrant pattern with a drop in the intensity signal, suggestive for the presence of variant DM1 expansions (Supplementary Fig. 1B, left panel).

Accordingly, "reverse" TP-PCR performed using either DM1/CC-F (Fig. 2c, right panel) and DM1/CCG-F (Supplementary Fig. 1B, right panel) revealed repeated, regular interruptions within the ladder peak only in these patients; in this regard, and in agreement with previous data [16], our results indicate that the proofreading activity of the polymerase would overcome in some cases the presence of few mismatches in the oligonucleotide sequences.

Sequence analysis of the forward TP-PCR products of pts 1-3 using an unlabeled DM1F primer documented a long run of pure CTG repeats without interruptions, similar to canonical DM1 patients (Supplementary Fig. 2A-C, left panels).

Conversely, sequence analysis of reverse TP-PCR products using an unlabeled DM1 R primer documented in these patients novel arrays of interruptions of the typical CTG stretch by CCG trinucleotides located in the distal $3^{\prime}$ end of the repeat (CCGCTGCTGCCG) (Supplementary Fig. 2): in particular, pt 1 had genotype (CTG) $)_{5}(\mathrm{CCGCTG}$ $\mathrm{CTG})_{46}, \quad$ Pt2 had genotype $(\mathrm{CTG})_{9}(\mathrm{CCGCTGCTG})_{61}$ (Supplementary Fig. 2A and B, right panels). Upstream of these regions, the sequences became uncertain $(\mathrm{CT} / \mathrm{CG})_{\mathrm{n}}$ in both patients, probably because of sequencing and PCR artifacts and/or possible somatic mosaicism [12].

Also in pt 3, sequence analysis confirmed an interrupted array, characterized by $(\mathrm{CTG})_{3}$ followed by uncertain $(\mathrm{CTG} / \mathrm{CCG})_{5}$ (Supplementary Fig. 2C, right panel).

Fluorescent-PCR for repeat size assessment

Fluorescent-PCR is currently used for the molecular diagnosis of genetic diseases associated with pathologically expanded alleles repeats $[21,22]$. This technique allows to assess the size of expanded alleles containing up to 320 triplet repeats, while the size of larger expanded alleles cannot be estimated [21, 22]. 


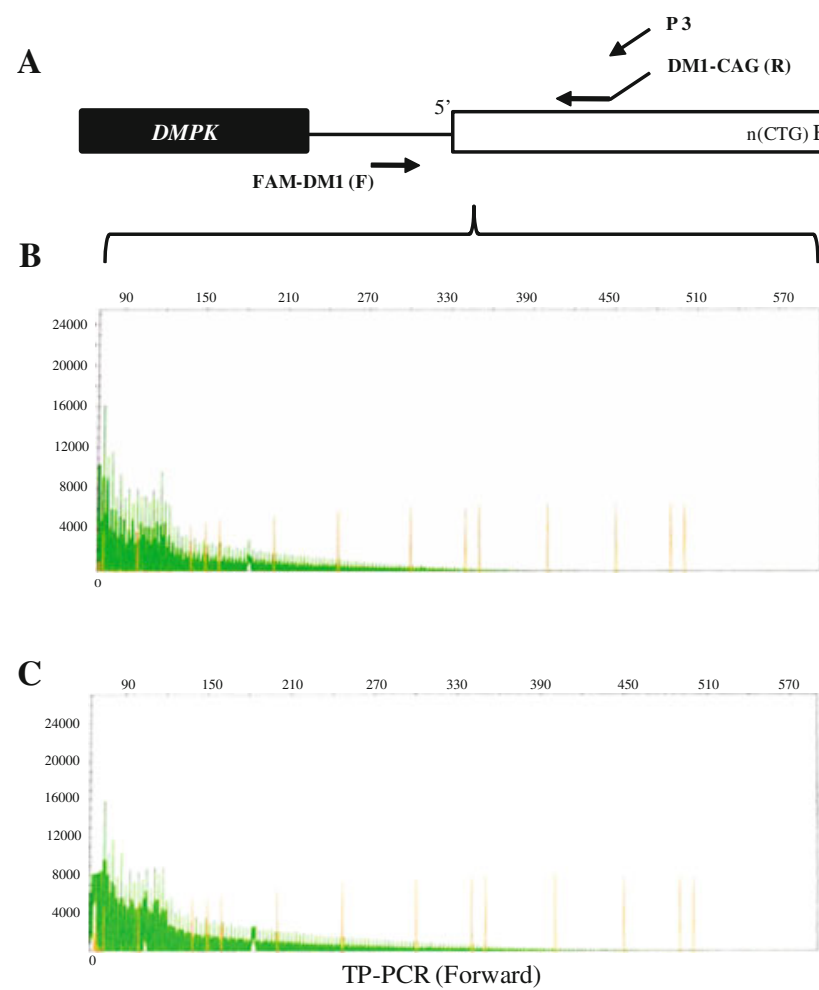

Fig. 2 Analysis of CTG repeats at the $D M P K$ locus by bidirectional TP-PCR. a Genomic organization around the DM1 expansion. Coding regions of DMPK and SIX5 are pictured as black boxes, CTG repeat region as white box and intronic sequences as thick black line. The PCR primers used in this study are indicated as arrows. DNA from patients was amplified using DM1/CAG R in the forward TP-PCR (green) and DM1/CC F in the reverse TP-PCR (blue). The yellow

By fluorescent PCR we were able to assess the size of the variant expanded allele in leukocytes DNA from pt 3, estimating an allele containing about 65 repeats (Fig. 3d) while, according to the sensitivity of this technique [21, 22], both pt 1 and pt 2 showed an expanded allele greater than 320 triplets (Fig. 3b, c). The size of variant expanded alleles in these two patients was then estimated by Southern blot analysis of fluorescent PCR products.

This analysis documented a smearing signal between 1.6 and $2.1 \mathrm{~kb}$ corresponding to alleles carrying 550-700 repeats in pt 1 , while pt 2 showed a smearing signal between 1.8 and $2.5 \mathrm{~kb}$ (600-830 repeats) (Fig. 3e). In pt 3 , sizing by Southern blot was comparable to that ascertained by capillary electrophoresis (one allele of $0.5 \mathrm{~kb}$ corresponding to about 65 repeats, Fig. 3d, e).

\section{Muscle RNA-FISH-immunofluorescence studies}

In DM1 pathology, the RNAs containing CUG expansions fold into hairpin structures and accumulate in nuclear foci, interfering with the activities of specific RNA-binding protein families, the muscleblind-like, which regulate the alternative splicing of several genes [28, 29].

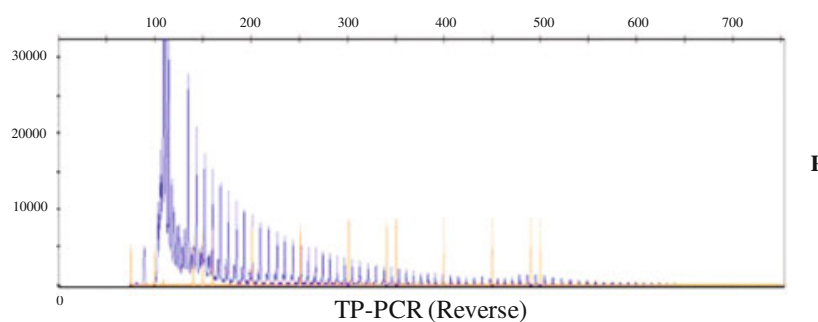

peaks correspond to the GS500 LIZ (Applied Biosystems, Foster City, USA) marker. b TP-PCR of a canonical DM1 patient. c TP-PCR of Pt1: the forward reaction documented pathologically expanded alleles with a pattern similar to the canonical DM1 patient; the electropherograms of the reverse TP-PCR displayed closely repeated gaps within the ladder peak. Similar data was obtained in pts 2 and 3 (data not shown)

To investigate if the presence of the CCG interruptions within the CTG array might lead to modifications in the higher structure of RNA transcripts and/or their interactions with RNA binding proteins, we performed RNA-FISHimmunofluorescence analysis with CAG probe and MBNL1 antibody on 6- $\mu \mathrm{m}$ muscle sections available from pts 1-3.

In all three patients studied, FISH using a CAG probe documented the presence of several ribonuclear inclusions per nucleus (Fig. 4a, d, g and k); MBNL1 immunofluorescence studies showed its co-localization with the ribonuclear foci, similar to what observed in a canonical DM1 patient (Fig. 4c, f, i and 1).

Alternative splicing analysis of developmentally regulated exons

To determine whether the presence of CCG interruptions would influence the aberrant alternative splicing pattern observed in DM1 tissues [24-27], we analyzed the splicing pattern of INSR, TNNT2, RYRland CLCN1 in muscle RNA from two controls, two canonical DM1 patients and pts 1-3 (Fig. 5). Indeed, all alternative splicing analyzed by densitometric analysis resulted similarly mis-regulated in pts 1-3 


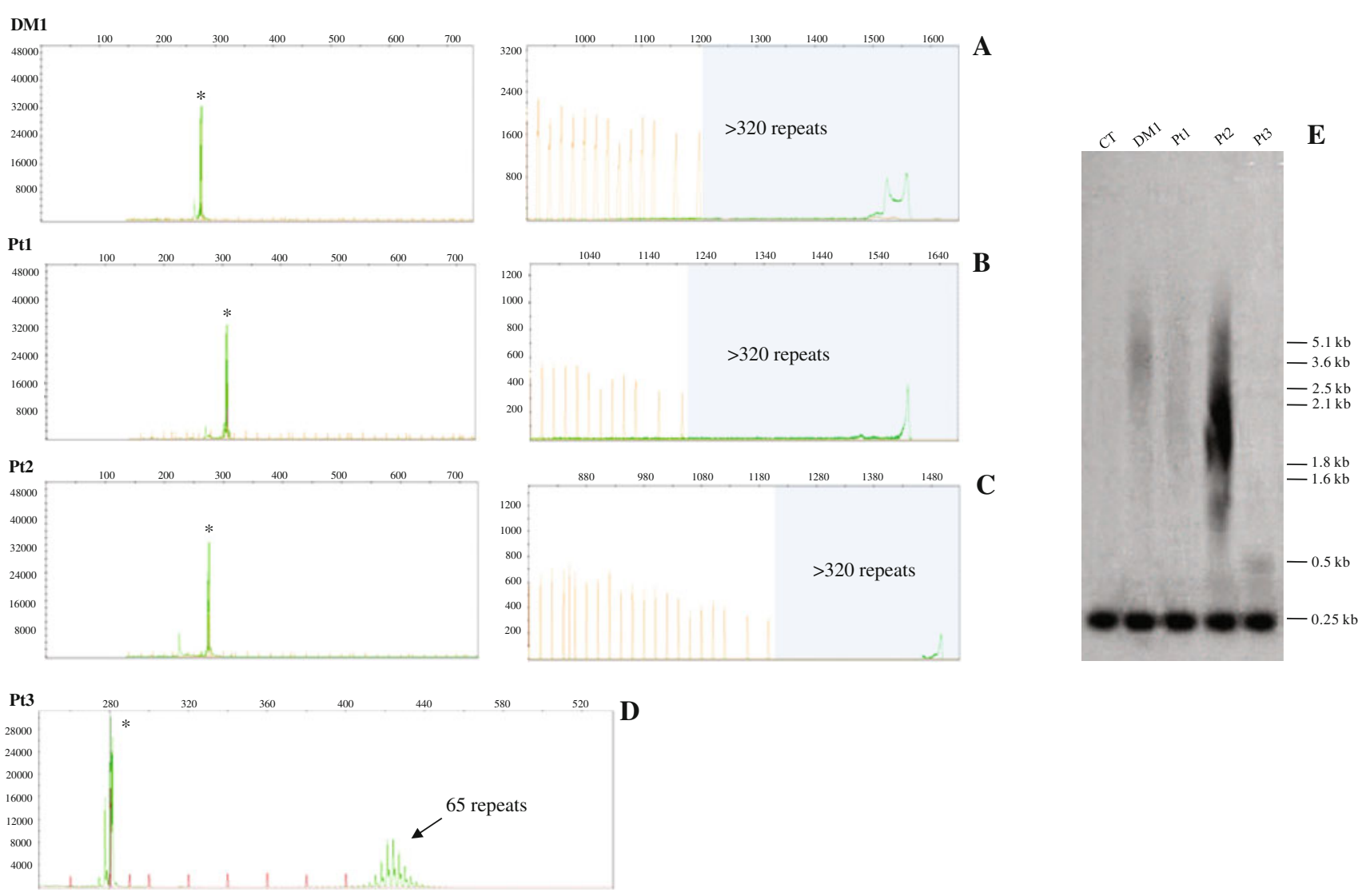

Fig. 3 Chromatograms of fluorescent PCR performed on leukocytes DNA from pts 1-3 and one DM1 patient. The yellow peaks correspond to the GS1200 LIZ (Applied Biosystems, Foster City, USA) marker. Asterisks indicate the wild-type allele (around $256 \mathrm{bp}$ ). The light blue square indicates the interval in which it is not possible to assess the size of expanded alleles. Canonical DM1 patient (a), Pt 1 (b) and Pt 2 (c) carry an allele containing $>320$ repeats; Pt 3 (d) has about 65 repeats. e Southern blot analysis of fluorescent PCR

as well as in two non-variant DM1 patients, showing a relative increase in the embryonic splice variants (Fig. 5).

Prevalence of variant repeats in DM1 families

By reverse TP-PCR, we screened 100 index cases of distinct genetically confirmed DM1 families diagnosed by LR $\mathrm{PCR} /$ Southern blot, to assess the prevalence of variant repeats in our cohort of study; this screening documented a pattern suggestive of variant repeats in 2 out of 100 patients tested (Pts4-5 of this study) (Fig. 6a, b).

Sequence analysis confirmed the presence of $\mathrm{CCG}$ interruptions also in these patients. However, in these cases the CCG repeats were more scattered along the CTG array compared to pts 1-3: in particular, pt 4 had genotype $(\mathrm{CTG})_{16} \mathrm{CCG}(\mathrm{CTG})_{4} \mathrm{CCG}(\mathrm{CTG})_{8} \mathrm{CCG}(\mathrm{CTG})_{5} \mathrm{CCG}(\mathrm{CTG})_{2}$ $\mathrm{CCG}(\mathrm{CTG})_{2} \mathrm{CCG}(\mathrm{CTG})_{8}$, while Pt5 had genotype $(\mathrm{CTG})_{12}$ $\mathrm{CCG}(\mathrm{CTG})_{6} \mathrm{CCG}(\mathrm{CTG})_{5} \mathrm{CCG}(\mathrm{CTG})_{5}$. Again, upstream of these regions, sequences became unreadable; moreover, products. Wild-type alleles show a molecular weight $(M W)$ of about $0.25 \mathrm{~kb}$. As an internal standard, a canonical DM1 patient shows a fully expanded allele with a range of 1,200-1,700 repeats (smear between 3.6 and $5.1 \mathrm{~kb}$ ). Pt 1 carries about 550-700 repeats (smear between 1.6 and $2.1 \mathrm{~kb}$ ), while in pt 2 the expansion contains about 600-830 repeats (smear between 1.8 and $2.5 \mathrm{~kb}$ ). In pt 3 , such a test confirms the result obtained with fluorescent PCR (band around $0.5 \mathrm{~kb}$, about 65 repeats)

sequence analysis of the forward TP-PCR products using an unlabeled DM1F primer documented also in these cases a pure CTG stretch (Supplementary Fig. 3). The size of the expanded alleles, estimated by LR PCR/Southern blot was about 900 repeats in pt 4 and 970 repeats in pt 5 (Fig. 1).

Overall we estimated a prevalence of $4.8 \%(5 / 103)$ variant expansions in our cohort of DM1 families.

\section{Discussion}

Expansion disorders are caused by a dynamic mutation (polymorphic short tandem repeats, interspersed within the gene sequences), arising through DNA replication and repair. Most frequently, pathological expansions involve trinucleotide repeats, including CAG, CTG, CGG and GAA within exons or in the untranslated gene regions [30].

Such polymorphic tracts are short and stable in healthy individuals, while in affected families the tracts are longer 


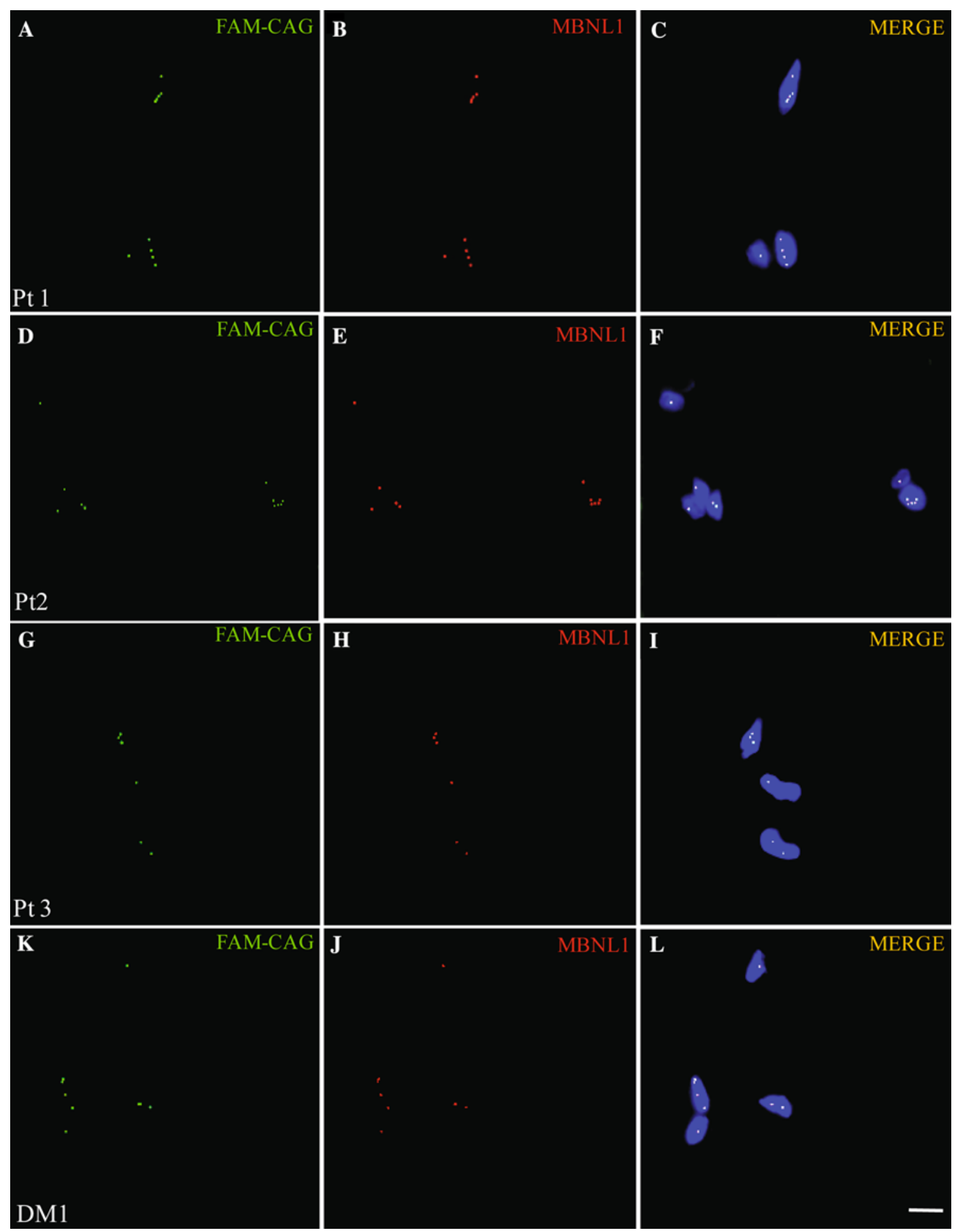

Fig. 4 RNA-Fluorescence in situ hybridization (RNA-FISH) and immunofluorescence (IF) studies for MBNL1 on muscle sections of Pt1-3 and a canonical DM1 patient. RNA-FISH using (CAG) 6 CA probe conjugated with $5^{\prime}$ fluorescein $(\mathrm{FAM})(\mathbf{a}, \mathbf{d}, \mathbf{g}$ and $\mathbf{k}$ panels,

and unstable. They have a strong tendency to expand rather than contract themselves during intergenerational and somatic divisions. green) combined with IF using MBNL1 monoclonal antibody HL 1822 [3A4-1E] (b, e, $\mathbf{h}$ and $\mathbf{j}$ panels, red) shows ribonuclear foci that co-localize with the signal for MBNL1 in muscle nuclei $(\mathbf{c}, \mathbf{f}, \mathbf{i}$ and I panels, nuclei counterstained in blue by DAPI). Scale bar $20 \mu \mathrm{m}$

In contrast to other triplet repeat disorders, in which the presence of interruptions within the expanded array are widely documented, the pathological expansion associated 
Fig. 5 Semi-quantitative RTPCR analysis on total RNA extracted from muscle biopsies of pts 1-3, two controls (CT) and two canonical DM1 patients (DM1); the $\beta$-actin signal was used as reference standard. Right panel scheme of the exon/ intron layout of human INSR, TNNT2, RYR1 and CLCN1.

Exons and introns are represented as thick and thin boxes respectively. Left panel; in pts 1-3 show an aberrant splicing of all these genes, similar to that found in the DM1 patients; histograms on top of each gel represent the relative amount of each aberrant premRNA specie, estimated as percentage values of the aberrant isoform versus its corresponding total pre-mRNA
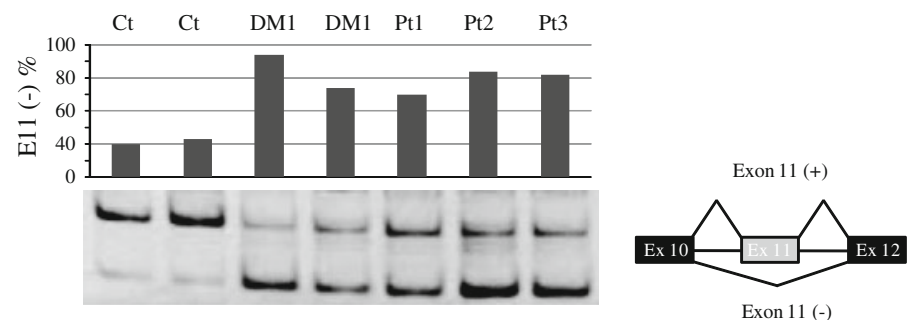

INSR
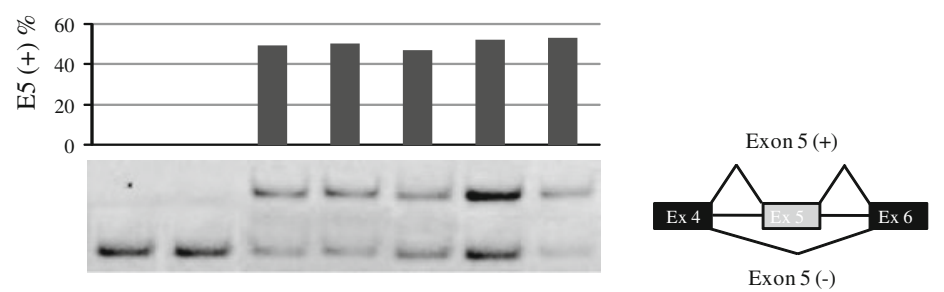

TNNT2
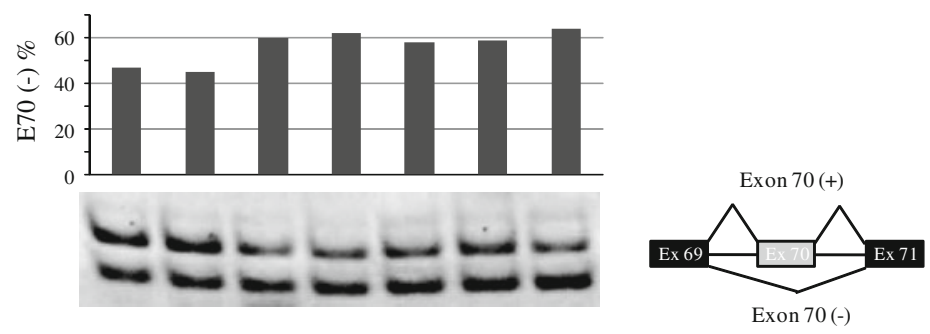

RYRI ASI
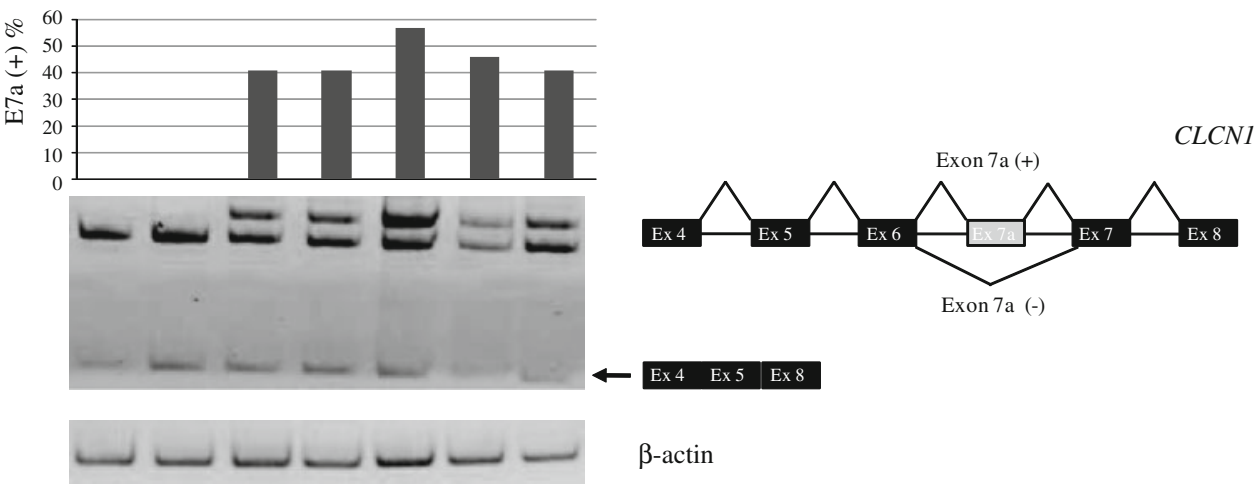

$\beta$-actin with DM1 has been assumed to be a pure CTG repeat [3, 5, 6]. The one exception to this was represented by a ' 37 ' repeat allele containing CCG interruptions identified in an anonymous sperm donor [31].

However, two research studies recently documented variant DM1 expanded alleles, characterized by the presence of CCG, CTC and/or GGC interruptions in the regular primary structure of the $3^{\prime}$-end of the CTG array, with a prevalence of $5 \%$ and $3-4 \%$ in their respective cohorts $[11,12]$.

The detection of variant DM1 alleles containing interruptions within the CTG array raised several important issues. First, the presence of GC interruptions at the $3^{\prime}$-end of the CTG array may lead to atypical (presence of gaps in the repeat primed-PCR ladder) or ambiguous (positive by
Southern blot analysis of genomic DNA, but negative by repeat primed PCR and/or amplification across the array) results at routine molecular testing, possibly leading to false negative results $[11,16]$.

Moreover, variant DM1 alleles frequently showed a relatively shorter size and they were also characterized by a greater inter-generational stability [11, 12]. Such evidence suggested that interruptions within the CTG array could have a stabilizing effect not only on the interrupted tract itself but also on the proximal, uninterrupted expanded CTG stretch, likely altering the binding of cis-elements that regulate the stability of the repeat [11].

Finally, the association of specific clinical features not typical of DM1 in some families carrying variant 
A

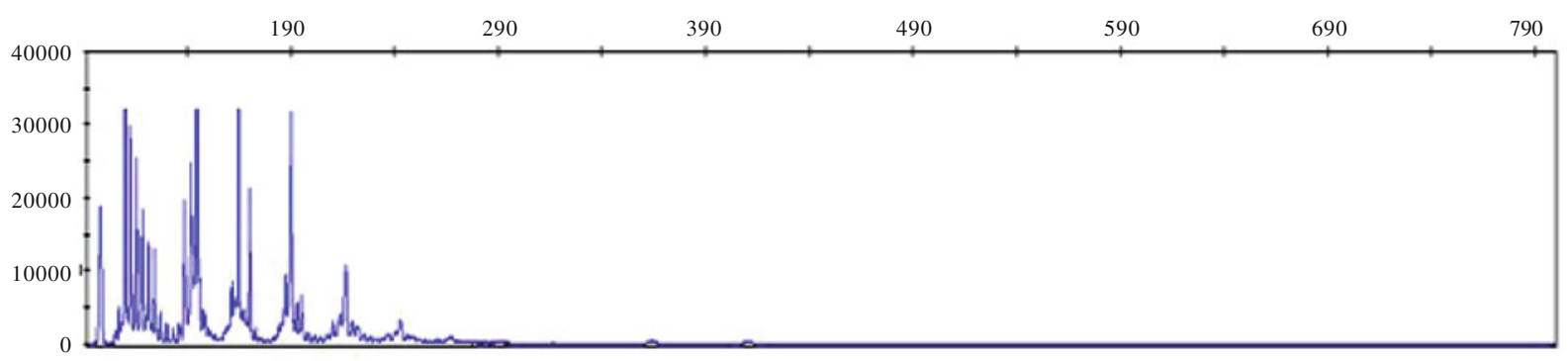

Pt 4

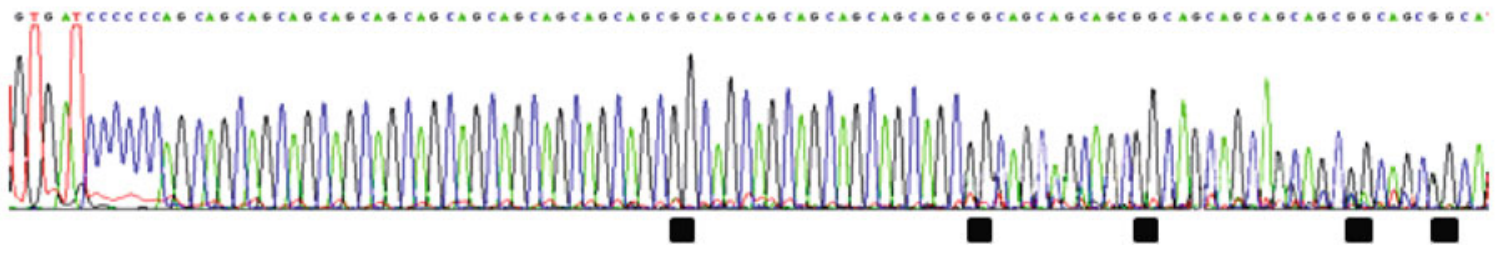

B
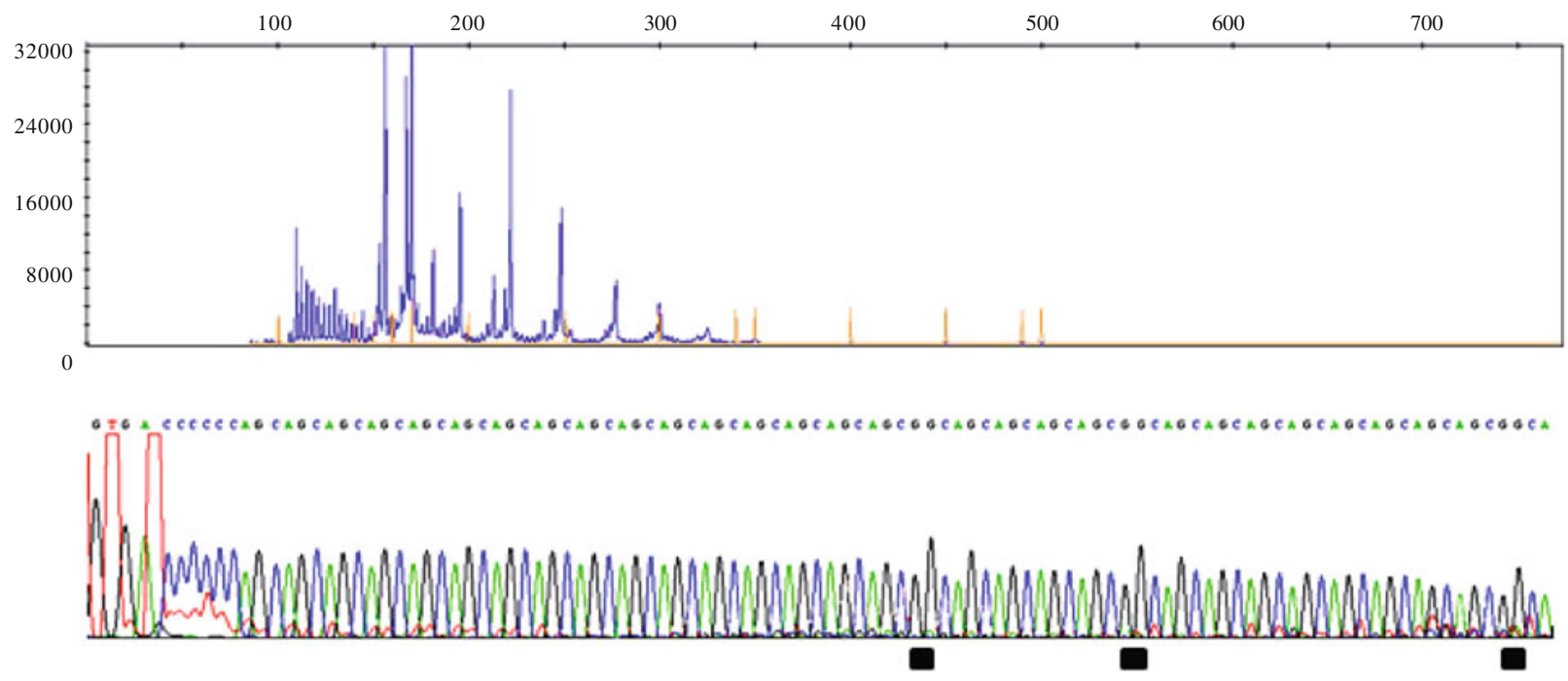

\section{- $\mathrm{CGG}$}

Fig. 6 Analysis of the $D M P K$ locus by reverse TP-PCR using oligo DM1/CC F. a The electropherogram of pt 4 shows several gaps in the fluorescent peak suggestive for variant expansions. Direct sequencing

expansions suggested that these interruptions might modulate the clinical phenotype, possibly affecting the mutational dynamics of the variant expansions within individual tissues, the formation of RNA foci and/or their interaction with splicing factors involved in DM1 pathogenesis [11, 12].

To clarify these issues, we searched for the presence of variant expansions in our cohort of patients clinically affected by Steinert's disease; the group of study included three patients repeatedly testing negative for DM1 and 100 index cases belonging to unrelated DM1 families previously diagnosed at our center by routine LR-PCR/Southern blotting [20]. of the reverse TP-PCR products using the antisense oligonucleotide (DM1 R) confirmed the presence of CCG interruptions (black boxes) in the typical CTG repeats. b Similar findings were obtained in Pt5

In agreement with previous studies, our results confirm that the presence of CCG interruptions at the $3^{\prime}$-end of the expanded CTG array at the DMPK locus can affect PCR amplification of the variant expansions, in some cases with a misleading negative result (Fig. 1).

Indeed, three of our five variant DM1 patients were apparently homozygous for a wild-type allele at routine DM1 molecular testing.

However, all of them showed both clinical (Table 1) and muscle morphological features (centralized nuclei, ring fibers and preferential type I fiber atrophy, data not shown), highly suggestive of DM1 [32]. 
Thus, we strongly reconsidered such a diagnosis. With regards to recent literature $[11,12]$ we searched for variant expansions using bidirectional TP-PCR; the forward reaction amplified a pathologically expanded allele in all of them, and the reverse reaction using modified oligonucleotides containing either CC (DM1/CC F) or CCG (DM1/ CCG F), showed discontinuous signals in the fluorescence peaks, likely caused by the presence of interruptions in the CTG array $[12,16]$.

Sequencing of TP-PCR products confirmed the presence of CCG interruptions at the $3^{\prime}$-end of the CTG array, while the CTG stretch resulted uninterrupted at its $5^{\prime}$-end (Fig. 2, Supplementary Fig. 2).

The application of the screening by reverse TP-PCR to our DM1 cohort, including 100 unrelated families, enabled us to identify two other patients carrying variant expansions. Also in these cases, sequence analysis confirmed the presence of CCG interruptions at the $3^{\prime}$-end of the array.

In all variant cases, we identified novel patterns of expanded arrays (Supplementary Fig. 2 and Fig. 6); intriguingly, our data would suggest that different gaps of CCG interruptions within the CTG array might influence the LR-PCR amplification of variant expanded DM1 alleles, possibly affecting their higher DNA structure. Indeed, LR-PCR failed to amplify expanded alleles of pts 1-3 (Fig. 1), characterized by regular, tightly spaced CCG interruptions (Supplementary Fig. 2), while it successfully amplified the expanded repeats in pts 4-5 (Fig. 1), showing more irregular and scattered CCG interruptions (Fig. 6).

Thus, regarding DM1 molecular diagnosis, a proposed diagnostic algorithm should consist of a short PCR to bridge the repeat, followed by TP-PCR in cases showing just one allele. If both tests are negative but the clinical suspicion of DM1 remains high, classic Southern blot on genomic DNA should be performed to definitively exclude the presence of a pathological expansion at the $D M P K$ locus [33].

We estimated a prevalence of $4.8 \%$ of variant expansions in our DM1 population, similar to two previous studies [11, 12]. Indeed, such estimation defines the lower limit in our population, since this screen would only be able to detect variant repeats that are reasonably close to either the $3^{\prime}$ - or $5^{\prime}$-end of the array, while small numbers of variant and/or variants further from either end of the array may be undetected [16].

Interestingly, two studies concerning large cohorts of DM1 parent-offspring pairs documented repeat contractions in 4-6\% of cases, a percentage very close to that estimated for variant expansions [34, 35]. Unfortunately, we did not have the chance of studying any first-degree, affected relatives of our variant DM1 patients, but the literature has shown that variant DM1 alleles have a greater intergenerational stability than uninterrupted ones, being even biased toward contraction [11, 12]. Therefore, in relation to genetic counseling, further studies are needed to clarify if repeat contractions occur only for variant DM1 alleles during intergenerational transmission.

Regarding the clinical phenotype associated with variant DM1 expansions, one of the families diagnosed by Braida et al. [11] co-segregated a complex neurological phenotype variably including an intermediate CMT neuropathy, early hearing loss and encephalopathic attacks. Such a complex phenotype is clearly distinct from that observed in other variant DM1 patients described until now [11, 12], as well as in our cases (Table 1). The peculiar phenotype observed in such a family [11] could be either related to the specific sequence and combination of repeat variants present on that particular DM1 mutated allele (the only being characterized by GGC variant repeats and a perfect 9 bp CGG/ CCG hairpin structure), or to the occurrence of a co-segregating mutation in the $\mathrm{MYH14}$ gene, which is closed to $D M P K$ and mutations of which have been recently described in a family manifesting a complex neuropathy phenotype [36].

Overall, our variant DM1 patients displayed a typical DM1 phenotype (Table 1). Muscle MRI findings were also comparable to those reported in DM1, showing in the three cases examined a predominant involvement of the leg and posterior thigh muscles [37, 38].

The only difference with canonical DM1 patients might be represented by the lack of cognitive involvement. Although it is known that an age-dependent cognitive decline mainly involving frontal and temporal functions can occur in DM1 patients [18, 39, 40], none of our variant DM1 patients, whose average age was 60.4 years, showed a significant cognitive impairment at neuropsychological examination (Table S1).

In this regard, it has been documented that DM1 tissues show a different degree of somatic mosaicism [41-43] and that either changes in the methylation status of the expanded allele [44] and/or binding of specific regulatory factors to cis-elements flanking the repeat may influence the somatic instability at the DMPK locus [45]. Therefore, we may speculate that the presence of CCG interruptions within the expanded CTG array could affect one of these mechanisms, eventually leading to an increased stability of the variant DM1 allele particularly in the brain tissue. Studies on larger cohorts of variant DM1 patients will be helpful to clarify this issue.

Concerning genotype-phenotype correlations (similar to the canonical DM1 patients), also in our "variant" DM1 patients the size of variant expansions in leukocytes correlated with the age at onset of symptoms. Pts 1 and 2 (harboring shorter expansions had the onset of symptoms in their 50s, while in pts 4 and 5 (carrying larger expanded alleles), the onset occurred during the third decade of life 
(Table 1). Pt 3 carried approximately 65 repeats in leukocytes and from the age of 30 years developed a progressive eyelids ptosis, but she manifested skeletal muscle weakness only in her late $60 \mathrm{~s}$.

Previous studies $[11,12]$ hypothesized that the presence of interruptions in the regular primary structure of the expanded CTG tract could lead to conformational changes in the higher structure of the RNA transcripts, eventually affecting their toxic gain of function.

To address this topic, we analyzed muscle biopsies available from three out of our five variant DM1 patients. RNA-FISH using a CAG probe, combined with immunofluorescence for MBNL1 protein revealed the presence of nuclear foci and their co-localization with MBNL1 (Fig. 4c, f, i and 1), similar to what documented in canonical DM1 patients [23]. Moreover, we documented an aberrant alternative splicing of developmentally regulated exons involved in DM1 pathogenesis [24-27], with no obvious differences between patients with and without variant expansions (Fig. 5).

Thus, our results indicate that the presence of CCG interruptions do not considerably affect the toxic gain of function of expanded RNAs [45, 46], at least in those patients carrying a pattern of DNA rearrangement similar to pts 1-3 (CCGCTGCTG). On the other hand, future muscle studies in DM1 patients carrying various patterns of interruptions will address if changes in the array of the variant repeat would differently affect the accumulation of RNA foci and/or their co-localization with MBNL1.

In summary, our study documents for the first time that variant DM1 patients do not appear to present with particularly unusual symptoms and that characteristic MBNL1 positive ribonuclear foci and splicing defects are also observed in their muscle tissues. Our data also suggests that the presence of CCG interruptions at the $3^{\prime}$-end of expanded $D M P K$ alleles might modulate the clinical phenotype by affecting the mutational dynamics of the variant repeats: in this regard, we now plan to analyze the somatic stability of variant DM1 expansions in fibroblasts cell cultures available from some of our patients.

Finally, this study confirms that bidirectional TP-PCR is very sensitive to detect "variant" DM1 expansions, and that this technique should be included in the routine diagnostic protocol used for DM1 testing [47].

Acknowledgments This work was supported by Grants from the Italian Ministry of Scientific Research (linea D1, 2011). We also acknowledge Dr. Glen W. McWilliams for kindly revising the English form of the manuscript.

Conflicts of interest All the authors deny any financial support and conflict of interest regarding this research activity.

\section{References}

1. Harper PS (2001) Myotonic Dystrophy, 3rd edn. WB Saunders, London

2. Machuca-Tzili L, Brook D, Hilton-Jones D (2005) Clinical and molecular aspects of the myotonic dystrophies. Muscle Nerve 32:1-18

3. Fu YH, Pizzuti A, Fenwick RG, King J, Rajnarayan S, Dunne PW, Dubel J, Nasser GA, Ashizawa T, de Jong P et al (1992) An unstable triplet repeat in a gene related to myotonic muscular dystrophy. Science 255:1256-1258

4. Harley HG, Rundle SA, Reardon W, Myring J, Crow S, Brook JD, Harper PS, Shaw DJ (1992) Unstable DNA sequence in myotonic dystrophy. Lancet 339:1125-1128

5. Mahadevan M, Tsilfidis C, Sabourin L, Shutler G, Amemiya C, Jansen G, Neville C, Narang M, Barceló J, O’Hoy K et al (1992) Myotonic dystrophy mutation: an unstable CTG repeat in the $3^{\prime}$ untranslated region of the gene. Science 255:1253-1255

6. Brook JD, McCurrach ME, Harley HG, Buckler AJ, Church D, Aburatani H, Hunter K, Stanton VP, Thirion JP, Hudson T et al (1992) Molecular basis of myotonic dystrophy: expansion of a trinucleotide (CTG) repeat at the $3^{\prime}$ end of a transcript encoding a protein kinase family member. Cell 68:799-808

7. Lavedan C, Hofmann-Radvanyi H, Shelbourne P, Rabes JP, Duros C, Savoy D, Dehaupas I, Luce S, Johnson K, Junien C (1993) Myotonic dystrophy: size- and sex-dependent dynamics of CTG meiotic instability, and somatic mosaicism. Am J Hum Genet 52:1164-1174

8. Monckton DG, Wong LJ, Ashizawa T, Caskey CT (1995) Somatic mosaicism, germline expansions, germline reversions and intergenerational reductions in myotonic dystrophy males: small pool PCR analyses. Hum Mol Genet 4:1-8

9. Martorell L, Monckton DG, Sanchez A, Lopez De Munain A, Baigetm M (2001) Frequency and stability of the myotonic dystrophy type 1 premutation. Neurology 56:328-335

10. López Castel A, Cleary JD, Pearson CE (2010) Repeat instability as the basis for human diseases and as a potential target for therapy. Nat Rev Mol Cell Biol 11:165-170

11. Braida C, Stefanatos RK, Adam B, Mahajan N, Smeets HJ, Niel F, Goizet C, Arveiler B, Koenig M, Lagier-Tourenne C et al (2010) Variant CCG and GGC repeats within the CTG expansion dramatically modify mutational dynamics and likely contribute toward unusual symptoms in some myotonic dystrophy type 1 patients. Hum Mol Genet 19:1399-1412

12. Musova Z, Mazanec R, Krepelova A, Ehler E, Vales J, Jaklova R, Prochazka T, Koukal P, Marikova T, Kraus J et al (2009) Highly unstable sequence interruptions of the CTG repeat in the myotonic dystrophy gene. Am J Med Genet 149A:1365-1374

13. Pearson CE, Eichler EE, Lorenzetti D, Kramer SF, Zoghbi HY, Nelson DL, Sinden RR (1998) Interruptions in the triplet repeats of SCA1 and FRAXA reduce the propensity and complexity of slipped strand DNA (S-DNA) formation. Biochemistry 37:2701-2708

14. Choudhry S, Mukerji M, Srivastava AK, Jain S, Brahmachari SK (2001) CAG repeat instability at SCA2 locus: anchoring CAA interruptions and linked single nucleotide polymorphisms. Hum Mol Genet 10:2437-2446

15. Warner JP, Barron LH, Goudie D, Kelly K, Dow D, Fitzpatrick DR, Brock DJ (1996) A general method for the detection of large CAG repeat expansions by fluorescent PCR. J Med Genet 33:1022-1026

16. Radvansky J, Ficek A, Minarik G, Palffy R, Kadasi L (2011) Effect of unexpected sequence interruptions to conventional PCR and repeat primed PCR in myotonic dystrophy type 1 testing. Diagn Mol Pathol 20:48-51 
17. Mathieu J, Boivin H, Meunier D, Gaudreault M, Bégin P (2001) Assessment of a disease-specific muscular impairment rating scale in myotonic dystrophy. Neurology 56:336-340

18. Modoni A, Silvestri G, Pomponi MG, Mangiola F, Tonali PA, Marra C (2004) Characterization of the pattern of cognitive impairment in myotonic dystrophy type 1. Arch Neurol 61:1943-1947

19. Dello Russo A, Mangiola F, Della Bella P, Nigro G, Melacini P, Bongiorni MG (2009) Risk of arrhythmias in myotonic dystrophy: trial design of the RAMYD study. J Cardiovasc Med 10:51-58

20. Gennarelli M, Pavoni M, Amicucci P, Novelli G, Dallapiccola B (1998) A single polymerase chain reaction-based protocol for detecting normal and expanded alleles in myotonic dystrophy. Diagn Mol Pathol 7:135-137

21. Filipovic-Sadic S, Sah S, Chen L, Krosting J, Sekinger E, Zhang W, Hagerman PJ, Stenzel TT, Hadd AG, Latham GJ et al (2010) A novel FMR1 PCR method for the routine detection of low abundance expanded alleles and full mutations in fragile $\mathrm{X}$ syndrome. Clin Chem 56:399-408

22. Chen L, Hadd A, Sah S, Filipovic-Sadic S, Krosting J, Sekinger E, Pan R, Hagerman PJ, Stenzel TT, Tassone F et al (2010) An information-rich CGG repeat primed PCR that detects the full range of fragile $\mathrm{X}$ expanded alleles and minimizes the need for southern blot analysis. J Mol Diagn 12:589-600

23. Santoro M, Modoni A, Masciullo M, Gidaro T, Broccolini A, Ricci E, Tonali PA, Silvestri G (2010) Analysis of MTMR1 expression and correlation with muscle pathological features in juvenile/adult onset myotonic dystrophy type 1 (DM1) and in myotonic dystrophy type 2 (DM2). Exp Mol Pathol 89:158-168

24. Savkur RS, Philips AV, Cooper TA (2001) Aberrant regulation of insulin receptor alternative splicing is associated with insulin resistance in myotonic dystrophy. Nat Genet 29:40-47

25. Philips AV, Timchenko LT, Cooper TA (1998) Disruption of splicing regulated by a CUG-binding protein in myotonic dystrophy. Science 280:737-741

26. Kimura T, Nakamori M, Lueck JD, Pouliquin P, Aoike F, Fujimura H, Dirksen RT, Takahashi MP, Dulhunty AF, Sakoda S (2005) Altered mRNA splicing of the skeletal muscle ryanodine receptor and sarcoplasmic/endoplasmic reticulum $\mathrm{Ca}^{2+}$-ATPase in myotonic dystrophy type 1. Hum Mol Genet 14:2189-2200

27. Charlet-B N, Savkur RS, Singh G, Philips AV, Grice EA, Cooper TA (2002) Loss of the muscle-specific chloride channel in type 1 myotonic dystrophy due to misregulated alternative splicing. Mol Cell 10:45-53

28. Miller JW, Urbinati CR, Teng-Umnuay P, Stenberg MG, Byrne BJ, Thornton CA, Swanson MS (2000) Recruitment of human muscleblind proteins to (CUG)(n) expansions associated with myotonic dystrophy. EMBO J 19:4439-4448

29. Mankodi A, Teng-Umnuay P, Krym M, Henderson D, Swanson M, Thornton CA (2003) Ribonuclear inclusions in skeletal muscle in myotonic dystrophy types 1 and 2. Ann Neurol 54:760-768

30. Eckert KA, Hile SE (2009) Every microsatellite is different: intrinsic DNA features dictate mutagenesis of common microsatellites present in the human genome. Mol Carcinog 48:379-388

31. Leeflang EP, Arnheim N (1995) A novel repeat structure at the myotonic dystrophy locus in a 37 repeat allele with unexpectedly high stability. Hum Mol Genet 4:135-136
32. Harper PS (1994) Myotonic dystrophies. In: Engel AG, FranziniArmstrong C (eds) Myology, 2nd edn. McGraw-Hill Inc., USA, pp 1192-1219

33. International Myotonic Dystrophy Consortium (IDMC) (2000) New nomenclature and DNA testing guidelines for myotonic dystrophy type I (DM1). Neurology 54:1218-1221

34. Harley HG, Rundle SA, MacMillan JC, Myring J, Brook JD, Crow S, Reardon W, Fenton I, Shaw DJ, Harper PS (1993) Size of the unstable CTG repeat sequence in relation to phenotype and parental transmission in myotonic dystrophy. Am J Hum Genet 52:1164-1174

35. Ashizawa T, Dubel JR, Dunne PW, Dunne CJ, Fu YH, Pizzuti A, Caskey CT, Boerwinkle E, Perryman MB, Epstein HF et al (1992) Anticipation in myotonic dystrophy. II. Complex relationships between clinical findings and structure of the GCT repeat. Neurology 42:1877-17833

36. Choi BO, Kang SH, Hyun YS, Kanwal S, Park SW, Koo H, Kim SB, Choi YC, Yoo JH, Kim JW et al (2011) A complex phenotype of peripheral neuropathy, myopathy, hoarseness, and hearing loss is linked to an autosomal dominant mutation in MYH14. Hum Mutat 32:669-677

37. Kornblum C, Lutterbey G, Bogdanow M, Kesper K, Schild H, Schröder R, Wattjes MP (2006) Distinct neuromuscular phenotypes in myotonic dystrophy types 1 and 2: a whole body highfield MRI study. J Neurol 253:753-761

38. Stramare R, Beltrame V, Dal Borgo R, Gallimberti L, Frigo AC, Pegoraro E, Angelini C, Rubaltelli L, Feltrin GP (2010) MRI in the assessment of muscular pathology: a comparison between limb-girdle muscular dystrophies, hyaline body myopathies and myotonic dystrophies. Radiol Med 115:585-599

39. Modoni A, Silvestri G, Vita MG, Quaranta D, Tonali PA, Marra C (2008) Cognitive impairment in myotonic dystrophy type 1 (DM1): a longitudinal follow-up study. J Neurol 255:1737-1742

40. Meola G, Sansone V, Perani D, Scarone S, Cappa S, Dragoni C, Cattaneo E, Cotelli M, Gobbo C, Fazio F et al (2003) Executive dysfunction and avoidant personality trait in myotonic dystrophy type 1 (DM-1) and in proximal myotonic myopathy (PROMM/ DM-2). Neuromuscul Disord 13:813-821

41. Anvret M, Ahlberg G, Grandell U, Hedberg B, Johnson K, Edstrom L (1993) Larger expansions of the CTG repeat in muscle compared to lymphocytes from patients with myotonic dystrophy. Hum Mol Genet 2:1397-1400

42. Ashizawa T, Dubel JR, Harati Y (1993) Somatic instability of CTG repeat in myotonic dystrophy. Neurology 43:2674-2678

43. Thornton CA, Johnson KJ, Moxley RT (1994) Myotonic dystrophy patients have larger CTG expansions in skeletal muscle than in leukocytes. Ann Neurol 35:104-107

44. López Castel A, Nakamori M, Tomé S, Chitayat D, Gourdon G, Thornton CA, Pearson CE (2011) Expanded CTG repeat demarcates a boundary for abnormal $\mathrm{CpG}$ methylation in myotonic dystrophy patient tissues. Hum Mol Genet 20:1-15

45. Cleary JD, Tomé S, López Castel A, Panigrahi GB, Foiry L, Hagerman KA, Sroka H, Chitayat D, Gourdon G, Pearson CE (2010) Tissue- and age-specific DNA replication patterns at the CTG/CAG-expanded human myotonic dystrophy type 1 locus. Nat Struct Mol Biol 17:1079-1087

46. Lee JE, Cooper TA (2009) Pathogenic mechanisms of myotonic dystrophy. Biochem Soc Trans 37:1281-1286

47. Prior TW (2009) Technical standards and guidelines for myotonic dystrophy type 1 testing. Genet Med 11:552-555 